\title{
Current Role and Importance of USG Guided FNAC for Rapid Diagnosis of Suspicious Mass Lesions
}

\author{
Maulik Jethva ${ }^{\oplus 1}$, Parth Dholakiya ${ }^{\circledR 2}$, Hiral Hapani ${ }^{\oplus 3}$ \\ ${ }^{1}$ Associate Professor, Department of Radio Diagnosis, PDU Medical College \& Govt. Hospital, Rajkot, Gujarat, India, ${ }^{2}$ Resident Doctor, Department of Radio Diagnosis, \\ PDU Medical College \& Govt. Hospital, Rajkot, Gujarat, India, ${ }^{3}$ Assistant Professor, Department of Radio Diagnosis, PDU Medical College \& Govt. Hospital, Rajkot, \\ Gujarat, India.
}

\section{Abstract}

Background: Ultrasound guided Fine needle aspiration is a diagnostic procedure that inserts a small needle into a suspicious mass to extract some cells which are then visualized under the microscope. The aim of the present study was to determine utility and safety of ultrasound guided FNAC in rapid evaluation of various mass lesions. Subjects and Methods: In this study, 120 patients of age between 20-60 years with various mass lesions referred for ultrasonography guided FNAC to the department of radiodiagnosis, PDU Medical College and Civil hospital, Rajkot, Gujarat over a period of 1.5 years from 1/5/2018 to 1/11/2019are included. Results: Ultrasonography guided FNAC was performed by the same investigator, an experienced radiologist, using a real time scanner (logicP9; GE Healthcare) under standard septic precautions. Conclusion: Our study contributes to the conclusion that USG guided FNAC can easilybe used for the aspiration of lesion of the breast, thyroid, lymph nodes, liver, lung, musculoskeletal system etc. It does not use ionizing radiation, has no known harmful effects, and provides rapid diagnosis of the suspicious mass lesions \&a firm diagnosis of impalpable lesions can be made preoperatively. Advantages of USG guided FNAC:- 1. Cost effectiveness, 2. No radiation hazards, 3. Easy to perform, 4. Dynamic evaluation of vessels, 5. Perfect real time Localization of the target lesion, 6. No hospitalization, 7. Least pre \& post procedure care.

Keywords: USG guided FNAC, Mass Lesions

Corresponding Author: Parth Dholakiya, Resident Doctor, Department of Radio Diagnosis, PDU Medical College \& Govt. Hospital, Rajkot, Gujarat, India.

E-mail: parth.dholakia28@gmail.com

Received: 25 January 2020

Revised: 22 February 2020

Accepted: 07 March 2020

Published: 05 July 2020

\section{Introduction}

In today's era with the help of various imaging modalities, an increasing number of mass lesions is detected and histological diagnosis is often necessary to determine the most appropriate management of these lesions.

In this clinical scenario, imaging-guided FNAC is one of the main methods to obtain tissue specimens. ${ }^{[1,2]}$

Various imaging techniques including computed tomography (CT) fluoroscopy and ultrasound (US) can be used to guide FNAC, but USG is most frequently used because of its cost effectiveness, real time imaging and without radiation hazard but in manycases, CT is preferred based on the localization of the lesion, artefacts or due to some patient-related factors.

Real-time US imaging allows for the dynamic evaluation of vessels and localization of target lesions thatmove during respiration.
In USG-guided FNAC the tip of the needle can be monitored throughout the procedure, and fine adjustments can be made quickly and precisely. ${ }^{[3,4]}$

USG guided FNAC is commonly used to obtain samples from the flowing sites:-

- Thyroid

- Breast

- Liver

- Lymph nodes

- Lung

- Mediastinum

\section{Aim and Objectives}

To assess the utility and safety of Ultrasound guidedFNAC in the evaluation of various mass lesions. 


\section{Subjects and Methods}

Sample size $=120$

Study design $=$ Retrospective study

Type of study $=$ Single center

Duration of study $=1 \mathrm{yr}$

Place of study=PDU Medical college $\&$ govt. hospital, Rajkot Consent for participation in study $=$ Yes.

\section{Patient Selection}

\section{Inclusion Criteria}

- All cases of mass lesions which are referred to our department $\&$ are accessible by ultrasound guidance.

\section{Exclusion Criteria}

- In the patients have risk factors like - moderate risk of bleeding, abnormal clotting function or thrombocytopenia etc.

\section{Machine Used}

GE LOGIQ P9 ultrasound machine.

Written consent for participation in the study is always be taken from the patient\& his/her relatives.

The indication and details of the procedure explained to patient.

Observations \& pathological results are recorded in patient proformas and analyzed statistically.

\section{Results}

Out of 120 patients ( $55 \%$ males, $45 \%$ females) of age group 20 to 60 years having mass lesions were biopsied using USG guidance.

Mass lesions of lung and liver were the most common sites.

Result of USG guided FNAC classified into three categories.

Diagnostic

Nondiagnostic descriptive

Inadequate

\section{Discussion}

USG guided FNAC is a collaborative work from radiologist and a pathologist. Some prerequisites before doing procedure should be like.

Prothrombin time (PT), activated partial thromboplastin time (APTT), and platelet count should be checked before percutaneous lung FNAC.
Table 1: The number of USG guided FNAC conducted by various sites were recorded out of total 120 patients

\begin{tabular}{lll}
\hline Name of system & No. of cases & Percentage \\
Lung & 35 & $29.20 \%$ \\
Liver & 30 & $25 \%$ \\
Lymph-node & 19 & $15.80 \%$ \\
Breast & 11 & $9.20 \%$ \\
Thyroid & 11 & $9.25 \%$ \\
Miscellaneous & 14 & $11.60 \%$ \\
\hline
\end{tabular}

Table 2: The outcomes of aspirated material are as follows

\begin{tabular}{|c|c|c|c|c|}
\hline System & Diagnos1 & Descriptir & Inadequate & Total \\
\hline lung & 32 & 2 & 1 & 35 \\
\hline liver & 26 & 3 & 1 & 30 \\
\hline $\begin{array}{l}\text { Lymph- } \\
\text { node }\end{array}$ & 19 & 0 & 0 & 19 \\
\hline thyroid & 10 & 1 & 0 & 11 \\
\hline breast & 11 & 0 & 0 & 30 \\
\hline miscellaneo & 12 & 1 & 1 & 14 \\
\hline Percentage & $91.66 \%$ & $5.83 \%$ & $3.33 \%$ & 120 \\
\hline
\end{tabular}

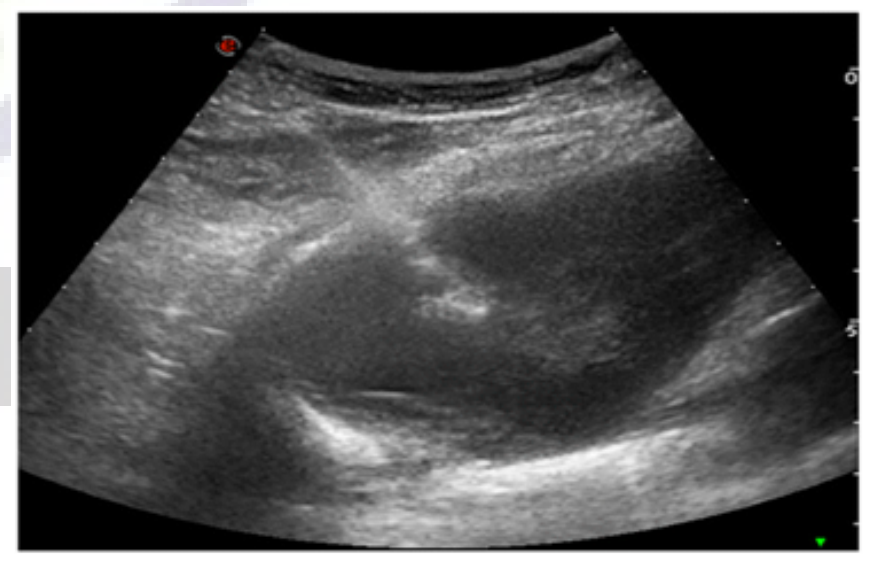

Figure 1: Ultrasound guided FNAC of a pulmonary nodule in the right lower lob

Oral anticoagulants should be stopped before a FNAC in accordance with the published guidelines on perioperative anticoagulation.

- Relative contraindications include:

- Platelet count $<100000 / \mathrm{ml}$

- APPT ratio or PT ratio >1.4

In these situations, a decision to proceed to FNAC should be made following discussion with a hematologist. 


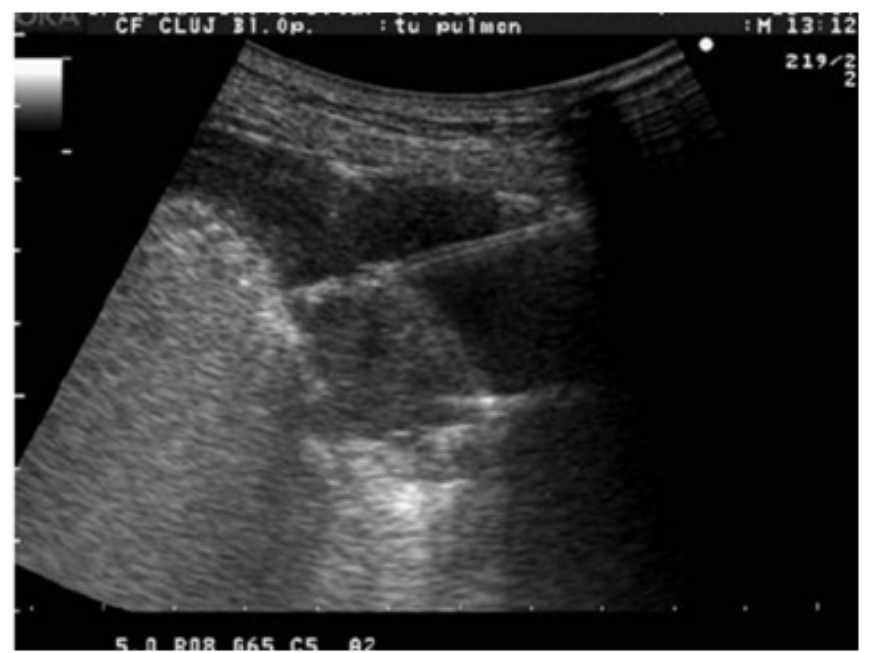

Figure 2: Ultrasound guided lung tumor FNAC. The needle passes over the pleural effusion penetrating the tumor

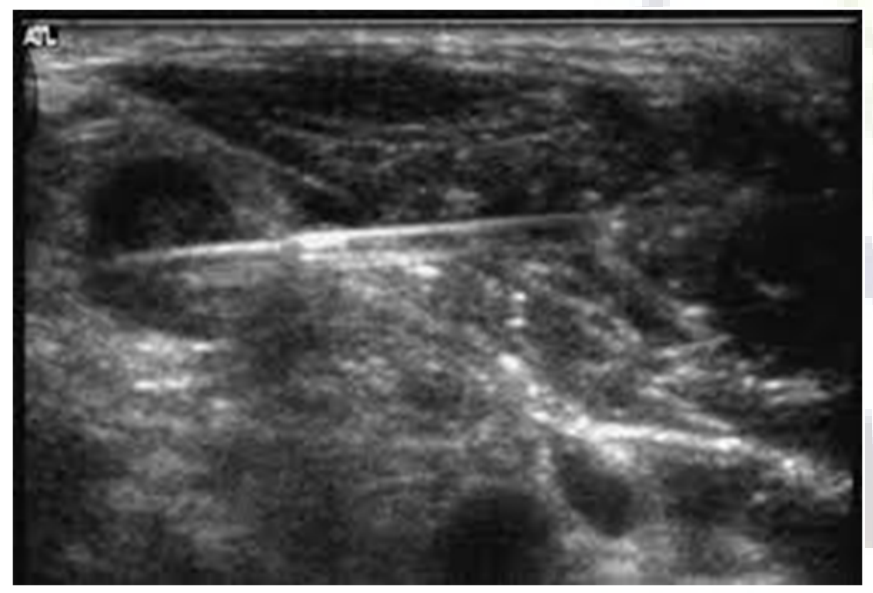

Figure 3: USG guided FNAC from neck node

In case of lesions of breast and thyroid even the lesions were palpable but specimen from suspicious area obtained with the help of USG guidance.

Choice of needle for the procedure is based on size and location of the lesion, intended needle trajectory, information expected from pathological sample and operator preference. ${ }^{[5]}$

Before procedure, atropine and pain relief medication should be given.

Patient position: patient can be positioned prone, supine or lying on side based on previously planned access site and needle trajectory.in case of lung FNAC when arms needed can be raised above head to widen the intercostal space.
For thyroid lesions, USG guided aspiration was initially recommended for lesions that were difficult to palpate, were predominantly cystic or had undergone a previously unsuccessful palpation guided biopsy / FNAC. ${ }^{[6]}$

A thyroid nodule detected by an imaging study having suspicious USG features (microcalcifications, local invasion, lymph node metastases, marked hypo echogenicity, irregular margins, solid composition, absence of a hypoechoic halo around the nodule, size $>1 \mathrm{~cm}$, taller than wide shape, and intra nodular vascularity) should be considered for USG guided FNAC.

USG guided breast FNAC has been developed to sample the impalpable breast lesions detected during breast screening.

In case of lymph nodes with the help of USG guidance aspirate can be obtained from specific active portion or a necrotic portion. ${ }^{[7]}$

USG guidance mainly used for impalpable lesions or when freehand biopsy performed was nondiagnostic. But in other lesions when USG guidance used it improved the yield of sample before specific area of interest can be obtained.

In case of abdomen and thoracic FNAC procedure, a good correlation between breathing movement and movement of needle should be obtained.

Some post procedure care applied like X-ray chest after thoracic FNAC procedure to look for pneumothorax, one-hour observation period, recording of pulse-bp etc.

Some complications can developed like pneumothorax in case of lung FNAC, injury to vessel, hematoma formation, spillage of tumor cells along the tract of needle.

Some disadvantages of USG guided FNAC includes, procedure becomes costly and tissue damage.

Follow up with histopathology reports done to know the adequacy of tissue samples or need of a Re-FNAC. ${ }^{[8]}$

\section{Conclusion}

We have done procedure on 120 patients having lesions of various systems with the help of USG guidance.

Our study contributes to the conclusion that USG guided FNAC has improved the yield of adequate sample and decreases the rate of re-FNAC. Innon-palpable lesions imaging guidance is definitely useful but even it has role in palpable lesion also when specific suspicious area of lesion is to be biopsied.

There are so many advantages of USG guided FNAC like, Cost effectiveness, real time imaging, no radiation hazard, easy to perform, dynamic evaluation of vessels, localization of target, no hospitalization etc. and there is no significant disadvantage of procedure. 
So nowadays role of USG guided FNAC is very important in diagnosis of suspicious mass lesions, we as a Radiologist always consider for it.

\section{References}

1. Erasmus RT, Zemlin AE. Clinical audit in the laboratory. J Clin Pathol. 2009;62(7):593-597. Available from: https://dx.doi.org/ 10.1136/jcp.2008.056929.

2. Jackson BR. Managing laboratory test use: Principles and tools. Clin Lab Med. 2007;27(4):733-781. Available from: https: //dx.doi.org/10.1016/j.cll.2007.07.009.

3. Rausch P, Nowels K, Jeffrey RB. Ultrasonographically guided thyroid biopsy: a review with emphasis on technique. J Ultrasound Med. 2001;20(1):79-85. Available from: https://dx. doi.org/10.7863/jum.2001.20.1.79.

4. Danese D, Sciacchitano S, Farsetti A, Andreoli M, Pontecorvi A. Diagnostic Accuracy of Conventional Versus SonographyGuided Fine-Needle Aspiration Biopsy of Thyroid Nodules. Thyroid. 1998;8(1):15-21. Available from: https://dx.doi.org/ 10.1089/thy.1998.8.15.

5. Kauf TL. The greater estimated expense associated with USGFNAB may still be a reasonable expenditure to achieve an additional correct diagnosis, but that is for individual practices and providers to decide. Head Neck. 2010;32(6):821-823. Available from: https://doi.org/10.1002/hed.21394.

6. Oz A, Demirkazik FB, Akpinar MG, Soygur I, Baykal A, Onder SC, et al. Efficiency of Ultrasound and Ultrasound-Guided Fine Needle Aspiration Cytology in Preoperative Assessment of
Axillary Lymph Node Metastases in Breast Cancer. J Breast Cancer. 2012;15(2):211-211. Available from: https://dx.doi.org/ 10.4048/jbc.2012.15.2.211.

7. Mainiero MB, Cinelli CM, Koelliker SL, Graves TA, Chung MA. Axillary Ultrasound and Fine-Needle Aspiration in the Preoperative Evaluation of the Breast Cancer Patient: An Algorithm Based on Tumor Size and Lymph Node Appearance. Am J Roentgenol . 2010;195(5):1261-1267. Available from: https://dx.doi.org/10.2214/ajr.10.4414.

8. Can A, Peker K. Comparison of palpation-versus ultrasoundguided fine-needle aspiration biopsies in the evaluation of thyroid nodules. BMC Research Notes. 2008;1(1):12-12. Available from: https://dx.doi.org/10.1186/1756-0500-1-12.

Copyright: (C) the author(s), 2020. It is an open-access article distributed under the terms of the Creative Commons Attribution License (CC BY 4.0), which permits authors to retain ownership of the copyright for their content, and allow anyone to download, reuse, reprint, modify, distribute and/or copy the content as long as the original authors and source are cited.

How to cite this article: Jethva M, Dholakiya $\mathrm{P}$, Hapani $\mathrm{H}$. Current Role and Importance of USG Guided FNAC for Rapid Diagnosis of Suspicious Mass Lesions. Asian J. Med. Radiol. Res. 2020;8(1):89-92.

DOI: dx.doi.org/10.47009/ajmrr.2020.8.1.16

Source of Support: Nil, Conflict of Interest: None declared.

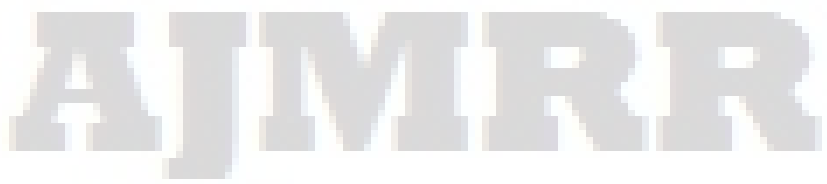

